\title{
Frequência das dermatoses nos pacientes da enfermaria de pediatria do Hospital Universitário Pedro Ernesto
}

\author{
Frequency of dermatoses in pediatric inpatients at \\ Hospital Universitário Pedro Ernesto

\section{Frecuencia de las dermatosis en los pacientes de la enfermeria del Hospital Universitario Pedro Ernesto}

\author{
Giane Pereira Giro Teixeira, ${ }^{*}$ Alexandre Carlos Gripp
}

\begin{abstract}
Resumo
Um total de 202 crianças, com idade entre 30 dias e 12 anos, internadas na enfermaria de pediatria do Hospital Universitário Pedro Ernesto foram examinadas por um período de doze meses. A prevalência das dermatoses foi de $83,67 \%$. As mais comuns (> 2\%) foram: nevos melanocíticos (20,29\%), mancha mongoliana (13,36\%), dermatite das fraldas (10,39\%), eczemátide (8,91\%), mancha café com leite $(7,92 \%)$, prurigo estrófulo $(7,42 \%)$, xerodermia (6,93\%), miliária (6,43\%), celulite $(5,44 \%)$, dermatite seborreica (4,95\%), nevo hipocrômico (3,96\%), dermatite atópica $(3,46 \%)$, dermatite de contato $(2,47 \%)$, alopecia difusa pós-quimioterapia $(2,47 \%)$. Houve variação na prevalência das dermatoses de acordo com a faixa etária analisada. Algumas afecções tiveram sua prevalência aumentada em decorrência da patologia apresentada pelos pacientes na internação.
\end{abstract}

Descritores: Dermatologia; Pediatria; Epidemiologia; Dermatoses.

\begin{abstract}
A total of 202 children aged 30 days to 12 years old were taken into the pediatric ward of HUPE. They were examined over a period of twelve months. The purpose of this study was to investigate the epidemiological characteristics of dermatoses in these children. The prevalence of skin lesions and diseases was $83,67 \%$. The most common ones (>2\%) were: pigmented nevus (20,29\%), mongolian spot (13,36\%), diaper dermatitis (10,39\%), pityriasis alba $(8,91 \%)$, café-au-lait spot $(7,92 \%)$, insect bites $(7,42 \%)$, xeroderma $(6,93 \%)$, miliaria $(6,43 \%)$, seborrheic dermatitis (4,95\%), cellulitis $(5,44 \%)$, nevus depigmentosus $(3,96 \%)$, atopic dermatitis $(3,46 \%)$, contact dermatitis $(2,47 \%)$, and diffuse alopecia after chemotherapy (2,47\%). There was variation in the prevalence of dermatoses according to the age group studied. Many diseases had higher frequency because of the patients' underlying conditions.
\end{abstract}

Keywords: Dermatology; Pediatrics; Epidemiology; Dermatoses.

\section{Resumen}

Un total de 202 niños, entre 30 días y 12 anos de edad, internados en la enfermería de pediatría del HUPE fueron examinados por un periodo de doce meses. La prevalencia de las dermatosis fue de $83,67 \%$. Las mas comunes (> $2 \%)$ fueron las siguientes: nevos melanocíticos $(20,29 \%)$, mancha mongólica $(13,36 \%)$, dermatitis de los pañales $(10,39 \%)$, eccematide $(8,91 \%)$, mancha café con leche $(7,92 \%)$, prurigo estrófulo $(7,42 \%)$, xeroderma $(6,93 \%)$, miliaria $(6,43 \%)$, celulitis $(5,44 \%)$, dermatitis seborreica $(4,95 \%)$, nevo hipocrómico $(3,96 \%)$, dermatitis atópica $(3,46 \%)$, dermatitis de contacto (2,47\%), alopecia difusa post-quimioterapia (2,47\%). Hubo variación en la prevalencia de las dermatosis de acuerdo con el grupo etáreo analizado. Algunas enfermedades aumentaron su prevalencia como consecuencia de la patología presentada por los pacientes en la internación.

Palabras clave: Dermatología; Pediatría; Epidemiología; Dermatosis. 


\section{Introdução}

\section{Dermatologia pediátrica - Histórico}

Dos ramos da medicina, a dermatologia é um dos mais antigos. É encontrada em relatos de cerca de cinco mil anos, além dos papiros egípcios de 1500 a.C., enquanto as coleções de Hipócrates da Grécia nos fornecem uma terminologia básica ainda em estudo.

Textos específicos de dermatologia, no entanto, são de origem muito recente. Dentre 213 dissertações inaugurais e 27 outros textos menores datados, entre os anos de 1573 e 1799, por George F. Still, pode-se encontrar apenas uma referência: Crusta Lactea Infantum, por Carol Strack, que foi obviamente dermatológica. ${ }^{1,2}$

O campo da dermatologia pediátrica tem tido reconhecimento significativo nos vários anos passados. Uma percepção de que a criança representa uma faceta do cuidado dermatológico especializado tem concordado com o rápido crescimento desta especialidade. ${ }^{3,4}$

\section{Prevalência de dermatoses}

A doença da pele representa entre 15 e 30\% da consulta pediátrica ambulatorial, ${ }^{5,6}$ e provavelmente uma grande proporção do trabalho do dermatologista envolve crianças.

Existe uma associação entre o nível socioeconômico e certas patologias cutâneas, especificamente as infectocontagiosas, assim como uma relação entre idade e afecções vinculadas à atopia.?

A impressão que se tem da frequência de dermatoses, baseada no atendimento de pacientes em ambulatórios, pode ser diferente da realidade, mostrada pelo censo epidemiológico de afecções cutâneas. Esses podem determinar com maior precisão a prevalência de dermatoses e a influência de certos fatores (biológicos, ecológicos e sociológicos) na ocorrência das afecções cutâneas.

Habitualmente, a frequência de cada dermatose tem sido relacionada ao número total de casos dermatológicos registrados em clínicas ambulatoriais. Assim sendo, o denominador é o número total de doentes atendidos e, portanto, uma amostra selecionada da população. Consequentemente, os índices relevantes tendem a ser maiores e, ademais, simplesmente indicam a frequência de uma dermatose em relação a outras afecções cutâneas, e não sua prevalência numa amostra populacional. No Brasil, encontramos na literatura relatos de apenas dois censos epidemiológicos. ${ }^{8}$

Há também na literatura vários trabalhos sobre a prevalência das dermatoses em crianças em consultas ambulatoriais, tanto de serviços públicos quanto de clínicas privadas.

Bechelli e colaboradores ${ }^{8}$ analisaram 5.150 escolares de 6 a 16 anos em Ribeirão Preto, São Paulo, e a prevalência de dermatoses foi de $72 \%$. As mais comuns estão apresentadas na tabela 1.

Pizzol ${ }^{9}$ relatou a incidência das dermatoses no atendimento ambulatorial no município de Viana, Espírito Santo, no ano de 1985. O atendimento dermatológico foi o responsável por 30,50\% das consultas, com 840 diagnósticos. Neste estudo, a alta incidência de piodermites e dermatozoonoses teve como fatores predisponentes o agrupamento

Tabela 1. Censo epidemiológico de lesões e afecções cutâneas em escolares de Ribeirão Preto, SP. Bechelli LM e cols., 1990.

\begin{tabular}{l|c}
\multicolumn{1}{c|}{ Tempo } & $\begin{array}{c}\text { Valor máximo da } \\
\text { normalidade }\end{array}$ \\
\hline Nevo pigmentado & $72,12 \%$ \\
Cicatrizes e lesões residuais & $36,02 \%$ \\
Efélides & $19,63 \%$ \\
Pediculose & $19,59 \%$ \\
Dermatite seborreica & $19,51 \%$ \\
Dermatoviroses & $18,58 \%$ \\
Acne & $15,73 \%$ \\
Lesões traumáticas & $9,50 \%$ \\
Pitiríase versicolor & $5,96 \%$ \\
Dermatofitose & $5,28 \%$ \\
Piodermites & $4,23 \%$ \\
Estrófulo + picada de inseto & $3,14 \%$ \\
Angioma & $3,01 \%$ \\
Nevo acrômico & $2,64 \%$ \\
Dermatite eczematosa & $2,58 \%$ \\
Ceratodermia palmoplantar & $2,10 \%$ \\
Desidrose & $2,04 \%$ \\
Víbices & $1,61 \%$ \\
Estomatite angular & $1,16 \%$ \\
Dermatoses actínicas & $1,13 \%$ \\
\hline & \\
&
\end{tabular}


excessivo das crianças em creches e o baixo nível cultural e socioeconômico da população estudada. As dermatoses mais encontradas estão demonstradas na tabela 2.

Nascimento ${ }^{10}$ e colaboradores realizaram o exame dermatológico em 289 recém-nascidos no berçário do Hospital Universitário Pedro Ernesto (HUPE), da Universidade do Estado do Rio de Janeiro (UERJ), durante períodos variáveis entre 1985 e 1989; as dermatoses mais frequentes foram listadas na tabela 3.

Mendonça e colaboradores ${ }^{11}$ examinaram 172 crianças em uma instituição dedicada ao cuidado de crianças abandonadas. O objetivo do trabalho foi determinar as dermatoses mais comuns numa população infantil que vive em um ambiente semifechado. Os grupos de dermatoses mais frequentes encontradas no estudo foram os apresentados na tabela 4.

Minelli e Minelli ${ }^{12}$ realizaram um estudo prospectivo de seis mil crianças em ambulatórios da rede pública e de clínica privada, entre 1978 e 1984, em Londrina, Paraná. As patologias mais frequentes foram agrupadas na tabela 5 .

Por meio desses dados, podemos observar que a prevalência das dermatoses varia muito

Tabela 2. Incidência das dermatoses em crianças de zero a seis anos no município de Viana, ES, no ano de 1985. Pizzol JL, 1988.

\begin{tabular}{l|c}
\hline \multicolumn{1}{c|}{ Dermatose } & Percentual \\
\hline Impetigo & $29,76 \%$ \\
Escabiose & $12,14 \%$ \\
Pediculose & $11,54 \%$ \\
Miliária & $5,59 \%$ \\
Perleche & $4,88 \%$ \\
Pitiríase alba & $3,57 \%$ \\
Ferimentos & $3,45 \%$ \\
Estrófulo & $3,45 \%$ \\
Eczema de contato & $3,21 \%$ \\
Varicela & $3,09 \%$ \\
Eczema atópico & $1,90 \%$ \\
Tungíase & $1,54 \%$ \\
Furunculose & $1,19 \%$ \\
Eczema retroauricular & $1,07 \%$ \\
Tinea corporis & $1,07 \%$ \\
\hline
\end{tabular}

de acordo com o grupo estudado: doenças infectocontagiosas prevalecendo em crianças de baixo nível socioeconômico, com maior frequência nas crianças em idade pré-escolar e escolar; dermatozoonoses mais comuns nos grupos de crianças em idade escolar, pela maior aglomeração que facilita sua transmissão.

Os neonatos têm algumas dermatoses próprias deste período, como mília, hiperplasia sebácea, eritema tóxico etc. Nos lactentes, há prevalência diferente de algumas afecções em relação aos outros grupos pediátricos. Através deste trabalho poderemos analisar as dermatoses na população pediátrica internada em um hospital, comparando-as com dados ambulatoriais e de censos epidemiológicos.

\section{Objetivos}

- Conhecer as características epidemiológicas que se apresentam nas crianças internadas na enfermaria geral de pediatria do HUPE;

- analisar, entre estas dermatoses, sua distribuição segundo a faixa etária;

Tabela 3. Prevalência das dermatoses em recémnascidos no berçário de um hospital universitário. Nascimento LV e cols., 1992.

\begin{tabular}{l|c}
\multicolumn{1}{c|}{ Dermatose } & Percentual \\
\hline Mancha mongólica & $77,50 \%$ \\
Mílio & $60,50 \%$ \\
Nevo vascular & $38,04 \%$ \\
Nevo nevocelular & $7,90 \%$ \\
Miliária & $2,40 \%$ \\
Eritema tóxico neonatal & $1,30 \%$ \\
Candidíase oral & $1,00 \%$ \\
Nevo azul & $1,00 \%$ \\
Onfalite & $0,60 \%$ \\
Acne neonatal & $0,60 \%$ \\
Eczema de contato & $0,60 \%$ \\
Eczema seborreico & $0,30 \%$ \\
Apêndice auricular & $0,30 \%$ \\
Nevo anêmico & $0,30 \%$ \\
Ostiofoliculite & $0,30 \%$ \\
Periporite & $0,30 \%$ \\
\hline
\end{tabular}


Tabela 4. Inquérito epidemiológico das dermatoses numa instituição infantil. Mendonça IRSM e cols., 1995.

\begin{tabular}{|c|c|c|}
\hline & Dermatose & Número absoluto \\
\hline \multirow[t]{2}{*}{ DERMATOZOONOSES } & Pediculose capitis & 45 \\
\hline & Escabiose & 14 \\
\hline \multirow[t]{2}{*}{ CERATODERMIAS } & Xerodermia & 30 \\
\hline & Ceratose Pilar & 2 \\
\hline \multirow[t]{4}{*}{ ECZEMAS } & Eczemátide & 26 \\
\hline & Eczema de contato & 2 \\
\hline & Eczema atópico & 2 \\
\hline & Eczema disidrótico & 1 \\
\hline \multirow[t]{2}{*}{ DISCROMIAS } & Mascular residuais & 24 \\
\hline & Nevo acrômico & 1 \\
\hline \multirow[t]{3}{*}{ PIODERMITES } & Furúnculo & 12 \\
\hline & Impetigo & 5 \\
\hline & Hordéolo & 4 \\
\hline \multirow[t]{9}{*}{ ONICOSES } & Distrofia ungueal & 5 \\
\hline & Unha em raquete & 3 \\
\hline & Braquioníquia & 3 \\
\hline & Microníquia & 2 \\
\hline & Pterigium unguea & 1 \\
\hline & Leuconíquia & 1 \\
\hline & Onicosquizia & 1 \\
\hline & Unhas pálidas & 1 \\
\hline & Ausência de lúnula & 1 \\
\hline \multirow[t]{2}{*}{ DERMATOVIROSES } & Molusco contagioso & 13 \\
\hline & Verruga vulgar & 3 \\
\hline \multirow[t]{2}{*}{ DERMATOMICOSES } & Tinea corporis & 8 \\
\hline & Tinea capitis & 5 \\
\hline
\end{tabular}

- avaliar em quantos pacientes a afecção cutânea foi a causa da internação.

\section{Casuística e métodos}

\section{Casuística}

Este trabalho foi realizado por um estudo prospectivo de 202 crianças internadas na enfermaria geral de pediatria do HUPE durante 12 meses.

A faixa etária variou de 30 dias a 12 anos (Fi- gura 1). Foi realizado o exame dermatológico (pele, cabelos, unhas e mucosas) nas 202 crianças, independentemente de relato da existência de alguma afecção cutânea. Pertenciam ao sexo masculino 116 pacientes, e ao sexo feminino, 86. Quanto à cor da pele, 74 eram brancos e 128 negros, incluídos aqui os considerados pardos (Figura 2).

\section{Métodos}

Os dados obtidos (nome, registro, idade, sexo, raça e patologias) foram anotados em fichas elaboradas para esta finalidade. A topografia da lesão 
Tabela 5. Dermatoses na infância: estudo estatístico de 6.000 casos. Minelli L, Minelli HJ, 1992

\begin{tabular}{|c|c|}
\hline Dermatose & Percentual \\
\hline Prurigo agudo & $12,70 \%$ \\
\hline Pitiríase alba & $6,86 \%$ \\
\hline Escabiose & $5,93 \%$ \\
\hline Impetignização & $4,55 \%$ \\
\hline Dermatite atópica & $4,36 \%$ \\
\hline Impetigo & $4,28 \%$ \\
\hline Eczematização & $4,08 \%$ \\
\hline Verruga vulgar & $4,03 \%$ \\
\hline Miliária & $3,38 \%$ \\
\hline Molusco contagioso & $3,31 \%$ \\
\hline Dermatite seborreica & $3,05 \%$ \\
\hline Tinha do couro cabeludo & $2,46 \%$ \\
\hline Ceratose pilar & $2,23 \%$ \\
\hline Fitofotomelanose & $2,01 \%$ \\
\hline Pitiríase versicolor & $1,91 \%$ \\
\hline Candidíase & $1,80 \%$ \\
\hline Dermatite de fraldas & $1,75 \%$ \\
\hline Tinha do corpo & $1,50 \%$ \\
\hline Eczema de contato & $1,48 \%$ \\
\hline Vitiligo & $1,48 \%$ \\
\hline Eczema microbiano & $1,46 \%$ \\
\hline Disidrose & $1,43 \%$ \\
\hline Urticária & $1,43 \%$ \\
\hline Nevo hipocrômico & $1,33 \%$ \\
\hline Larva migrans & $1,30 \%$ \\
\hline
\end{tabular}

também foi anotada em ficha especial.

O diagnóstico foi predominantemente clínico. Em alguns raros casos praticou-se exame micológico e histopatológico. Quando houve dúvida no diagnóstico, fez-se novo exame com acompanhamento do orientador.

As doenças foram agrupadas conforme suas afinidades etiológicas e seus aspectos clínicos. As doenças que apareceram mais raramente ou não cabiam dentro de uma classificação já utilizada foram agrupadas em tabela especial (miscelânea). Foram desconsideradas as lesões de hiper/hipocromia residual.
Crianças com afecção cutânea receberam, quando necessário, orientação e tratamento adequados.

\section{Resultados}

O total de diagnósticos foi de 290 em 169 pacientes. Das crianças examinadas, 33 (11,3\%) apresentaram o exame normal. Os diagnósticos agrupados segundo o grupo de doenças são descritos na figura 4. Não foram consideradas como diagnóstico as lesões hipocrômicas nem hipercrômicas residuais.

Dentre as causas de internação, a doença dermatológica foi responsável em 27 (13,36\%) dos pacientes, sendo mais comuns as piodermites. Algumas doenças tiveram grande variação na incidência de acordo com a faixa etária, sendo selecionadas e apresentadas discriminando os grupos separadamente nas figuras 3 e 4 .

Em relação à topografia das lesões, os resultados estão apresentados na figura 3. Os resultados das dermatoses mais prevalentes por grupo de doença estão relacionados nas tabelas de 5 a 17 . As dermatoses que apresentaram variação importante quanto à faixa etária estão apresentadas nas figuras 5 e 6.

A distribuição das dermatoses por grupo de doenças está descrita na tabela 18. As dermatoses mais frequentes na enfermaria de pediatria do HUPE estão descritas na tabela 19.

\section{Discussão}

Neste estudo, foram examinados aleatoriamente os pacientes internados, independentemente de relato de afecção cutânea, aproximando-se de um censo epidemiológico. O exame foi normal em $16,33 \%$ dos casos, um número bem menor que o encontrado na literatura. No entanto, houve influência de fatores que aumentaram a frequência de algumas dermatoses quando comparada à população geral:

- houve um aumento de casos das dermatoses que levam à internação, como piodermites, colagenoses, vasculites e outras;

- os pacientes internados por patologias que cursam com imunossupressão (Sida, neoplasias e/ou tratamentos quimio- 


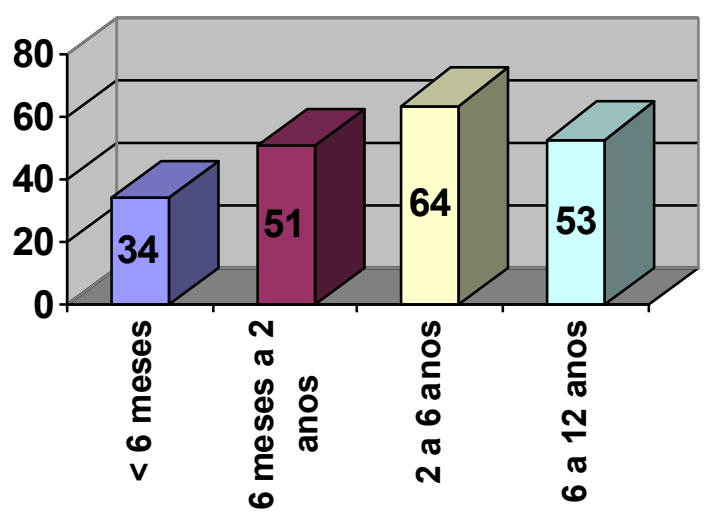

Figura 1. Distribuição dos pacientes segundo a faixa etária.

terápicos) provocaram um aumento na frequência da candidíase oral (1,98\%), herpes-zóster $(0,9 \%)$ e alopecia difusa $(2,4 \%)$ como consequência do tratamento;

- $\quad$ os doentes com coagulopatias, como nas leucemias e linfomas, frequentemente apresentavam petéquias e equimoses (1,48\% cada), que não são observadas comumente nas crianças normais.

O pequeno número de casos (202) para um estudo estatístico não contribuiu para uma avaliação mais fidedigna, principalmente das afecções mais raras. Discutiremos os resultados com número de afecções mais expressivo, que permitem melhor análise dos dados.

Pela grande variação de apresentação quanto à idade, algumas doenças tiveram mudanças de acordo com o subgrupo analisado e foram referidas nas figuras 5 e 6 .

A mancha mongoliana, que é extremamente frequente nos neonatos $(77,5 \%)$, principalmente os de raça negra, e com o avançar do tempo esmaece gradualmente, manifestou este comportamento também neste trabalho: esteve presente em 13,36\% dos casos. Obviamente, este número seria bem maior se a média de idade dos pacientes fosse menor.

A dermatite das fraldas foi a mais comum do grupo dos eczemas. É dermatose frequente nos dois primeiros anos de vida, criada pela imposição do uso das fraldas. A oclusão com umidade e maceração pela retenção de urina e fezes causam irritação da pele. Sua incidência é reduzida com o tempo, pelo abandono do uso das fraldas. A pre-

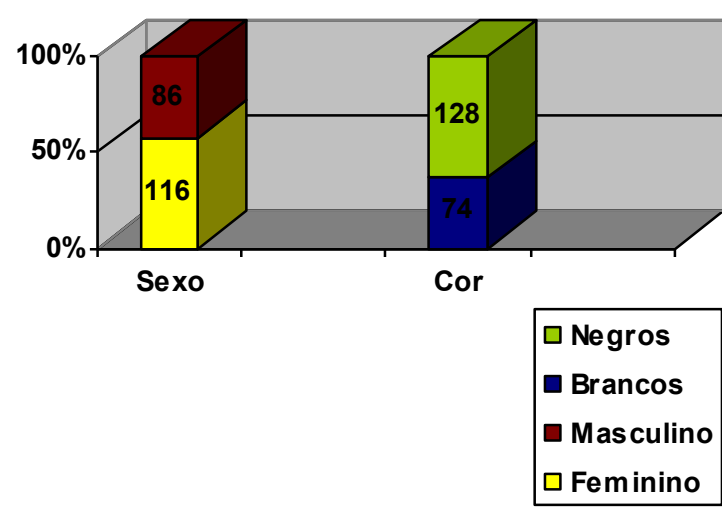

Figura 2. Distribuição dos pacientes por sexo e cor.

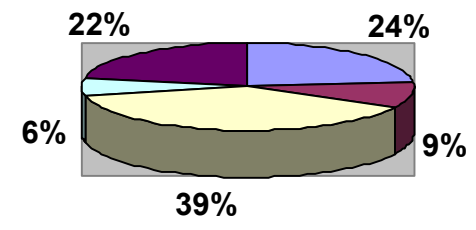

\begin{tabular}{|ll|}
$\square$ Cabeça & $\square$ Membros superiores \\
$\square$ Tronco & $\square$ Região pélvica \\
$\square$ Membros inferiores & \\
\hline
\end{tabular}

Figura 3. Distribuição das lesões quanto a topografia.

valência total foi de 10,39\% e destes $95,31 \%$ eram menores de dois anos. A eczemátide foi o segundo maior diagnóstico neste grupo, representando $8,91 \%$ dos doentes, e comumente se apresentava nas crianças com história pessoal ou familiar de atopia. Sua frequência na literatura é maior entre os seis a doze anos, o que também observamos. $\mathrm{O}$ eczema seborreico (4,95\%) é uma erupção eritematoescamosa que geralmente se desenvolve nos doze primeiros meses de vida, começando como descamação no couro cabeludo (crosta láctea). Foi diagnosticado apenas nos menores de dois anos, e predominantemente nos menores de seis meses. Os casos de dermatite de contato eram, invariavelmente, ao uso de esparadrapos. Dos pacientes com dermatite atópica (3,46\%), 42,8\% estavam internados pela extensão das lesões e infecção secundária.

Os nevos melanocíticos representaram o diagnóstico mais comum: 20,29\%. Não foi possível diferenciar entre os congênitos e adquiridos pela dificuldade dos pais de avaliar o tempo de surgimento da lesão. O número maior de lesões 


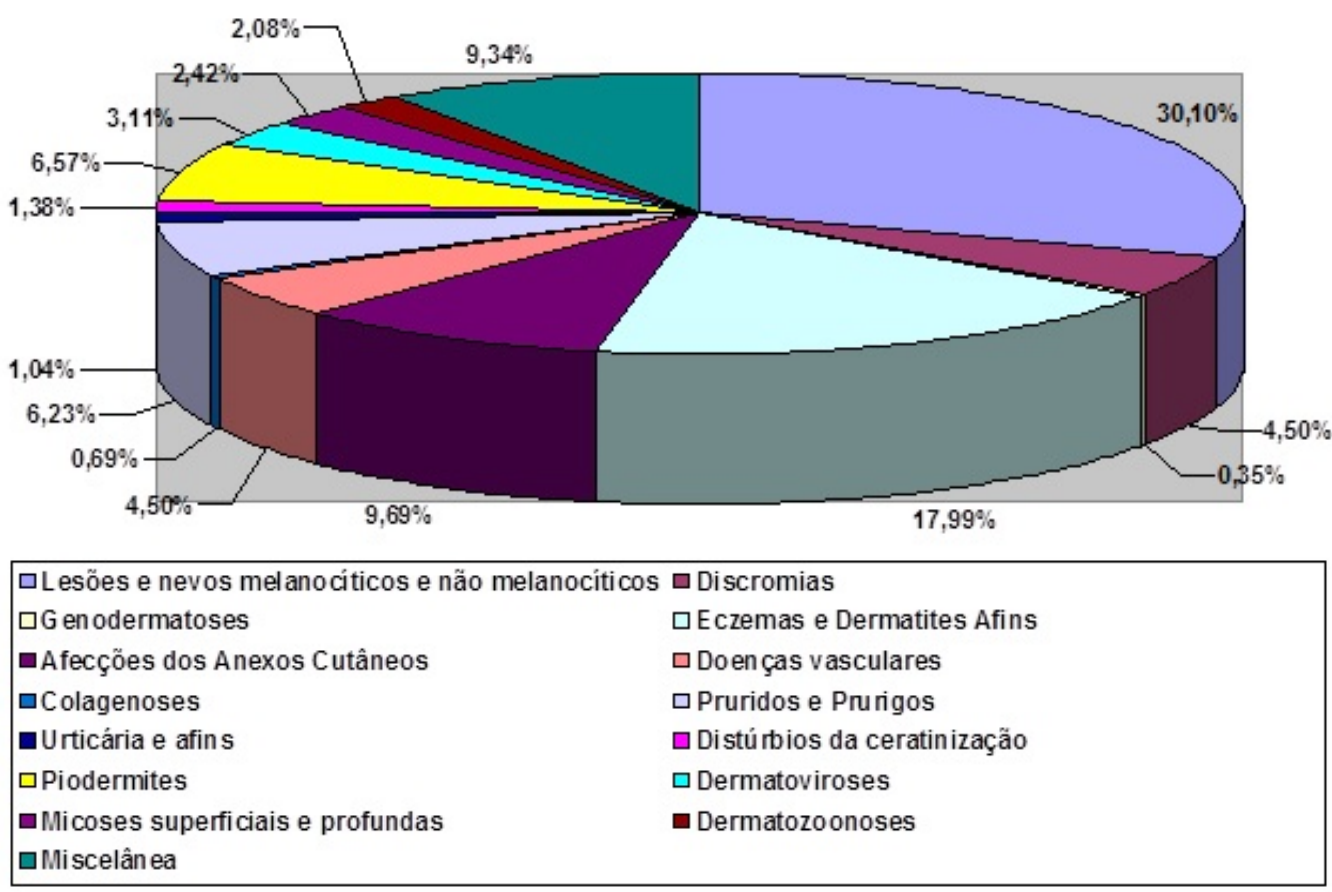

Figura 4. Distribuição das dermatoses por grupo de doenças.

Tabela 5. Distribuição das dermatoses por grupo de doenças. Lesões e nevos melanocíticos e não melanocíticos $(n=87)$.

\begin{tabular}{l|r}
\multicolumn{1}{c|}{ Dermatose } & Número absoluto (\%) \\
\hline Nevo melanocítico & $41(20,29 \%)$ \\
Mancha mongoliana & $27(13,36 \%)$ \\
Mancha café com leite & $27(13,36 \%)$ \\
Nevus Spillus & $1(0,49 \%)$ \\
Nevo de Becker & $1(0,49 \%)$ \\
Nevo de Ito & $1(0,49 \%)$ \\
\hline
\end{tabular}

Tabela 6. Distribuição das dermatoses por grupo de doenças. Eczemas e dermatites afins $(n=52)$.

\begin{tabular}{l|r}
\multicolumn{1}{c|}{ Dermatose } & Número absoluto (\%) \\
\hline Dermatite das fraldas & $21(10,39 \%)$ \\
Eczemátide & $18(8,91 \%)$ \\
Dermatite seborreica & $10(4,95 \%)$ \\
Dermatite atópica & $7(3,46 \%)$ \\
Dermatite de contato & $5(2,47 \%)$ \\
Eczema desidrótico & $1(0,49 \%)$ \\
\hline
\end{tabular}

Tabela 7. Distribuição das dermatoses por grupo de doenças. Afecções dos anexos cutâneos (n=28).

\begin{tabular}{l|rr} 
& Dermatose & Número absoluto $(\%)$ \\
\hline HIDROSES & Miliária & $13(6,43 \%)$ \\
TRICOSES & Alopecia difusa pós-quimioterapia & $5(2,47 \%)$ \\
ONICOSES & Hipertricose & $1(0,49 \%)$ \\
& Leuconíquia pontuada & $4(1,98 \%)$ \\
& Sulcos de Beau & $2(0,99 \%)$ \\
& Onicoatrofia & $1(0,49 \%)$ \\
& Unhas em vidro de relógio & $1(0,49 \%)$ \\
& Depressões cupuliformes & $1(0,49 \%)$ \\
\hline
\end{tabular}


Tabela 8. Distribuição das dermatoses por grupo de doenças. Piodermites $(n=19)$.

\begin{tabular}{l|r}
\multicolumn{1}{c|}{ Dermatose } & Número absoluto (\%) \\
\hline Celulite & $7(3,46 \%)$ \\
Abcesso & $4(1,98 \%)$ \\
Piomiosite & $3(1,48 \%)$ \\
Impetigo & $2(0,99 \%)$ \\
Foliculite & $2(0,99 \%)$ \\
Ectima & $1(0,49 \%)$ \\
\hline
\end{tabular}

Tabela 9. Distribuição das dermatoses por grupo de doenças. Pruridos e prurigos $(n=18)$.

\begin{tabular}{l|r}
\hline \multicolumn{1}{c|}{ Dermatose } & Número absoluto (\%) \\
\hline Prurigo estrófulo & $15(7,42 \%)$ \\
Prurido por xerose da pele & $3(1,48 \%)$ \\
\hline
\end{tabular}

Tabela 10. Distribuição das dermatoses por grupo de doenças. Discromias $(n=13)$.

\begin{tabular}{l|r}
\hline \multicolumn{1}{c|}{ Dermatose } & Número absoluto (\%) \\
\hline Nevo hipocrômico & $8(3,96 \%)$ \\
Efélides & $4(1,98 \%)$ \\
Vitiligo & $1(0,49 \%)$ \\
\hline
\end{tabular}

Tabela 11. Distribuição das dermatoses por grupo de doenças. Doenças vasculares $(n=13)$.

\begin{tabular}{l|r}
\hline \multicolumn{1}{c|}{ Dermatose } & Número absoluto (\%) \\
\hline Equimoses & $3(1,48 \%)$ \\
Petéquias & $3(1,48 \%)$ \\
$\begin{array}{l}\text { Púrpura de Henoch } \\
\text { Shoenlein }\end{array}$ & $2(0,99 \%)$ \\
Hemangioma plano & $2(0,99 \%)$ \\
Granuloma piogênico & $2(0,99 \%)$ \\
Livedo reticular fisiológico & $1(0,49 \%)$ \\
Vasculite séptica por & $1(0,49 \%)$ \\
\hline
\end{tabular}

Tabela 12. Distribuição das dermatoses por grupo de doenças. Dermatoviroses $(n=9)$.

\begin{tabular}{l|r}
\multicolumn{1}{c|}{ Dermatose } & Número absoluto (\%) \\
\hline Molusco contagioso & $3(1,48 \%)$ \\
Condiloma vulvar & $2(0,99 \%)$ \\
Herpes-zóster & $2(0,99 \%)$ \\
Verruga vulgar & $1(0,49 \%)$ \\
Varicela & $1(0,49 \%)$ \\
\hline
\end{tabular}

Tabela 13. Distribuição das dermatoses por grupo de doenças. Micoses superficiais e profundas $(n=8)$.

\begin{tabular}{l|r}
\hline \multicolumn{1}{c|}{ Dermatose } & Número absoluto (\%) \\
\hline $\begin{array}{l}\text { Candidiase oral } \\
\text { Paracoccidioidomicose } \\
\text { juvenil }\end{array}$ & $4(1,98 \%)$ \\
Pitiriase versicolor & $1(0,49 \%)$ \\
Tinea pedis interdigital & $2(0,99 \%)$ \\
\hline
\end{tabular}

Tabela 14. Distribuição das dermatoses por grupo de doenças. Dermatozoonoses $(n=6)$.

\begin{tabular}{l|r}
\multicolumn{1}{c|}{ Dermatose } & Número absoluto (\%) \\
\hline Escabiose & $3(1,48 \%)$ \\
Pediculose & $3(1,48 \%)$ \\
\hline
\end{tabular}

Tabela 15. Distribuição das dermatoses por grupo de doenças. Disturbios da ceratinização $(n=4)$.

\begin{tabular}{l|r}
\multicolumn{1}{c|}{ Dermatose } & Número absoluto (\%) \\
\hline Ceratose folicular & $3(1,48 \%)$ \\
Liquen espinuloso & $1(0,49 \%)$ \\
\hline
\end{tabular}

Tabela 16. Distribuição das dermatoses por grupo de doenças. Urticária e afins $(n=3)$.

\begin{tabular}{l|r}
\multicolumn{1}{c|}{ Dermatose } & Número absoluto (\%) \\
\hline Urticária & $2(0,99 \%)$ \\
Dermografismo & $1(0,49 \%)$ \\
\hline
\end{tabular}


Tabela 17. Distribuição das dermatoses por grupo de doenças. Colagenoses $(n=2)$.

\begin{tabular}{l|r}
\multicolumn{1}{c|}{ Dermatose } & Número absoluto (\%) \\
\hline Esclerodermia & $1(0,49 \%)$ \\
Dermatomiosite & $1(0,49 \%)$ \\
\hline
\end{tabular}

Tabela 18. Distribuição das dermatoses por grupo de doenças. Miscelânea $(n=28)$.

\begin{tabular}{l|r}
\hline \multicolumn{1}{c|}{ Dermatose } & Número absoluto (\%) \\
\hline Xerodermia & $14(6,93 \%)$ \\
Calosidade & $2(0,99 \%)$ \\
Intertrigo & $2(0,99 \%)$ \\
Cabelos descorados & $2(0,99 \%)$ \\
Língua fissurada & $1(0,49 \%)$ \\
Queilite angular & $1(0,49 \%)$ \\
Hiperplasia sebácea do & $1(0,49 \%)$ \\
recém-nato & $1(0,49 \%)$ \\
Doença de Letterer-Siwe & $1(0,49 \%)$ \\
Síndrome de Behçet & $1(0,49 \%)$ \\
Glossite losângica mediana & $1(0,49 \%)$ \\
\hline Esclerose tuberosa &
\end{tabular}

nos pré-escolares e escolares (Figura 3) mostra o aparecimento dos nevos melanocíticos adquiridos com o avançar da idade. No censo epidemiológico em escolares o número destas lesões chegou a 72,12\% das crianças. Já quando examinados recémnascidos, o número cai para apenas 7,9\%.

As piodermites em dados ambulatoriais respondem por 4 a $29 \%$ das doenças cutâneas na criança, com impetigo e furúnculo sendo os mais comuns. ${ }^{12}$ Verificamos, no entanto, predomínio das celulites e abscessos; certamente por causa da amostra estudada. Houve uma curva ascendente em relação aos subgrupos etários (Figura 5), confirmando a maior prevalência em pré-escolares e escolares. Em todos os casos de celulite nos menores de dois anos, a lesão estava situada na face.

As lesões residuais não foram consideradas como diagnóstico, mas foram observadas em 33 pacientes e, como visto na figura 4, é mais vista nos pré-escolares e escolares, que são mais expostos a pequenos traumatismos e escoriações.
Tabela 19. Dermatoses mais frequentes na enfermaria da pediatria do HUPE.

\begin{tabular}{|c|c|}
\hline Dermatose & Número absoluto (\%) \\
\hline Nevos melanocíticos & $41(20,29 \%)$ \\
\hline Mancha mongoliana & $27(13,36 \%)$ \\
\hline Dermatite das fraldas & $21(10,39 \%)$ \\
\hline Eczemátide & $18(8,91 \%)$ \\
\hline Mancha café com leite & $16(7,92 \%)$ \\
\hline Prurigo estrófulo & $15(7,42 \%)$ \\
\hline Xerodermia & $14(6,93 \%)$ \\
\hline Miliária & $13(6,43 \%)$ \\
\hline Dermatite seborreica & $10(4,95 \%)$ \\
\hline Nevo hipocrômico & $8(3,96 \%)$ \\
\hline Dermatite atópica & $7(3,46 \%)$ \\
\hline Celulite & $7(3,46 \%)$ \\
\hline Dermatite de contato & $5(2,47 \%)$ \\
\hline Alopecia pós-quimioterapia & $5(2,47 \%)$ \\
\hline Leuconíquia pontuada & $4(1,98 \%)$ \\
\hline Abcesso & $4(1,98 \%)$ \\
\hline Candidíase oral & $4(1,98 \%)$ \\
\hline Efélides & $4(1,98 \%)$ \\
\hline Molusco contagioso & $3(1,48 \%)$ \\
\hline Escabiose & $3(1,48 \%)$ \\
\hline Pediculose & $3(1,48 \%)$ \\
\hline Piomiosite & $3(1,48 \%)$ \\
\hline Ceratose folicular & $3(1,48 \%)$ \\
\hline Equimoses & $3(1,48 \%)$ \\
\hline Petéquias & $3(1,48 \%)$ \\
\hline Prurido por xerose da pele & $3(1,48 \%)$ \\
\hline Púrpura de Henoch-Schoenlein & $2(0,99 \%)$ \\
\hline Hemangioma plano & $2(0,99 \%)$ \\
\hline Granuloma piogênico & $2(0,99 \%)$ \\
\hline Sulco de Beau & $2(0,99 \%)$ \\
\hline Urticária & $2(0,99 \%)$ \\
\hline Impetigo & $2(0,99 \%)$ \\
\hline Foliculite & $2(0,99 \%)$ \\
\hline Calosidade & $2(0,99 \%)$ \\
\hline Intertrigo & $2(0,99 \%)$ \\
\hline Cabelos descorados & $2(0,99 \%)$ \\
\hline Condiloma anal & $2(0,99 \%)$ \\
\hline Herpes-zóster & $1(0,49 \%)$ \\
\hline Nevus Spillus & $1(0,49 \%)$ \\
\hline Nevo de Becker & $1(0,49 \%)$ \\
\hline Nevo de Ito & $1(0,49 \%)$ \\
\hline Esclerose tuberosa & $1(0,49 \%)$ \\
\hline Desidrose & $1(0,49 \%)$ \\
\hline Hipertricose & $1(0,49 \%)$ \\
\hline Unha em vidro de relógio & $1(0,49 \%)$ \\
\hline Onicoatrofia & $1(0,49 \%)$ \\
\hline Depressões cupuliformes & $1(0,49 \%)$ \\
\hline
\end{tabular}




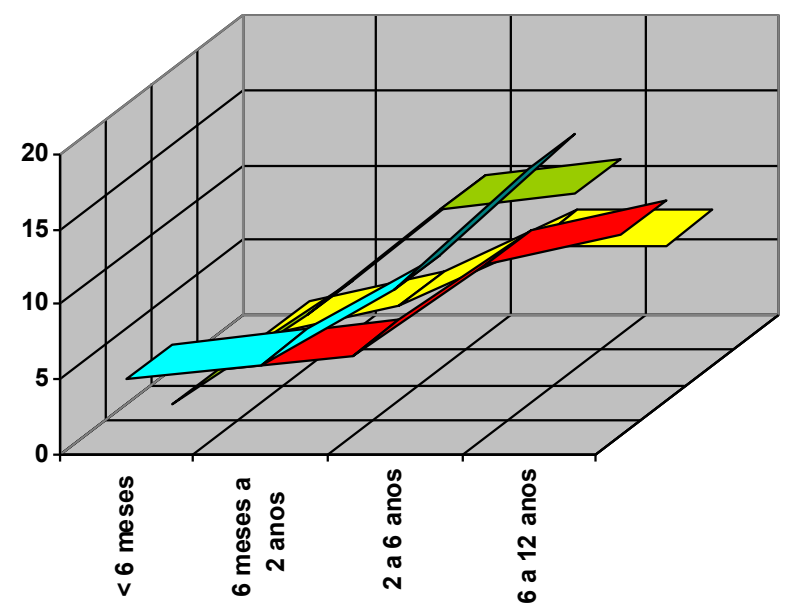

$\square$ Nevos melanocíticos $\square$ Lesões residuais $\square$ Piodermites $\square$ Eczematide

Figura 5. Aumento da prevalência das dermatoses em relação a idade dos pacientes.

Encontramos 14 casos de xerodermia (6,93\%). Optamos por relacioná-los no quadro de miscelânea, já que alguns pacientes apresentaram história de atopia, outros algum grau de desnutrição ou, em alguns, associação com os hábitos da criança (número de banhos, uso de sabões, temperatura da água), sendo que estes fatores foram encontrados inter-relacionados, o que dificultou a classificação dentro dos grupos de dermatoses.

Dentre as dermatoviroses a verruga vulgar lidera as estatísticas na literatura..$^{13}$ A criança é mais susceptível às viroses que os adultos pela menor oportunidade de contatos antigênicos, estimuladores da produção de anticorpos. O molusco contagioso foi mais frequente (1,48\%). A incidência aumentada de condiloma acuminado se deve à internação de crianças vítimas de abuso sexual.

A pediculose teve frequência menor quando comparada a censos epidemiológicos. Isto pode ter ocorrido pela faixa etária, que era predominantemente menor de seis anos (73,76\%), ao contrário das referências analisadas; com menor exposição a aglomerações que as tornassem susceptíveis. Por isso, poderíamos esperar também uma menor prevalência de piodermites, mas, como discutimos, pela necessidade de internação houve um aumento de casos.

O estrófulo $(7,4 \%)$ é quadro comum no primeiro e no segundo ano de vida. É uma reação de hipersensibilidade a diversos agentes. Os alérgenos mais comuns parecem ser toxinas de picada

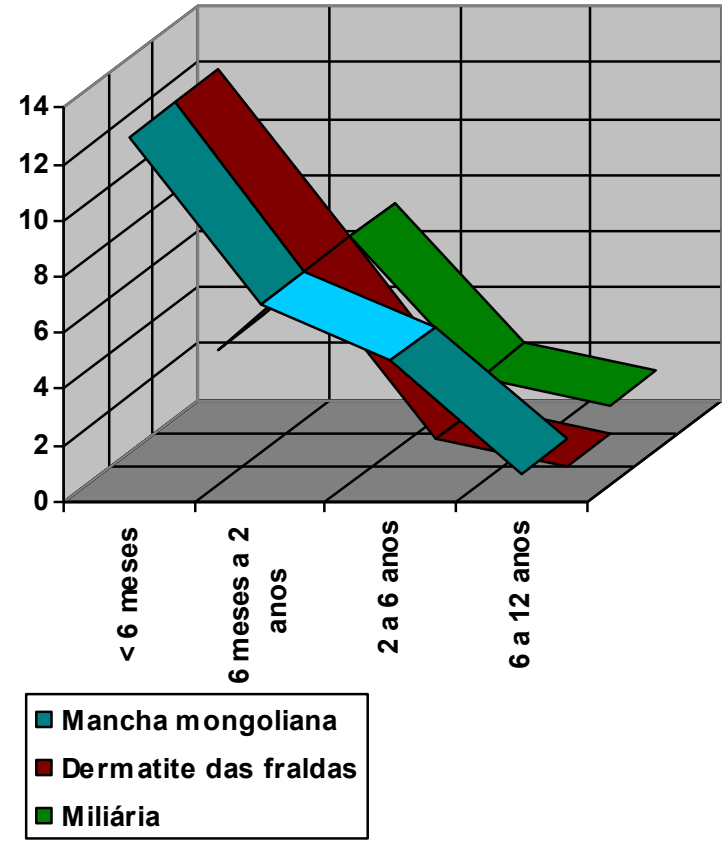

Figura 6. Dinimuição da prevalência das dermatoses em relação a idade dos pacientes.

de inseto. Foi raramente observado nas crianças acima de seis anos (apenas 0,9\%, provavelmente pelo desenvolvimento de tolerância à picada de inseto). A miliária esteve presente em $6,4 \%$ dos casos, e, destes, 76,9\% ocorreram em menores de dois anos. A enfermaria tinha boa ventilação (ar-condicionado), mas talvez o uso excessivo de roupas nas crianças menores tenha contribuído para este número. 
As manchas café com leite têm relatos de frequência bastante variáveis, desde 1 até 23\%, em um estudo com crianças escolares brancas. Em todos os casos vistos (7,92\%), os pacientes tinham uma única lesão, geralmente nos membros inferiores.

O nevo hipocrômico, nevo acrômico ou nevus despigmentosus $(3,96 \%)$ foi associado em uma única paciente com a esclerose tuberosa. Nos demais era lesão única, congênita, sem outros sintomas nem sinais associados. Sua incidência geral é de 1 a $3 \%{ }^{8}$

Algumas dermatoses, apesar de pouco vistas, destacaram-se pela apresentação nas crianças imunossuprimidas: os casos de herpes-zóster e candidíase oral eram em crianças em tratamento quimioterápico para leucemias e linfomas ou HIV positivas. Das que faziam quimioterapia, cinco desenvolveram alopecia difusa. Dos casos de molusco contagioso, apenas um (do total de três) era em paciente com Sida.

Dermatofitoses e pitiríase versicolor tiveram pequeno número de casos (apenas três pacientes no total, ou 1,48\%), o que é compatível com dados ambulatoriais de crianças menores de seis anos (1\%), a maior parte da nossa amostra, mas bem menor quando comparado às crianças maiores de seis anos (10 a 15\%).

Como já discutimos, as demais dermatoses, por seu número reduzido de casos vistos (menos de $2 \%$ ) dentro da pequena amostra, não nos fornece dados suficientes para uma melhor análise.

A população estudada de pacientes internados em um hospital público, que atende às classes com nível socioeconômico mais baixo, nos fez pensar em encontrar uma grande prevalência das dermatoses mais ligadas a esta condição.

Nas referências de atendimento ambulatorial, ${ }^{6}$ as doenças infectoparasitárias são mais comuns entre a população menos favorecida. No entanto, no censo epidemiológico em Ribeirão Preto, ${ }^{8}$ a pediculose capitis e a eczemátide tiveram realmente diferenças estatisticamente significantes entre as diferentes classes; enquanto as dermatoviroses e as piodermites, ao contrário do que se imagina, não têm diferença significativa entre estes níveis.

Algumas afecções frequentes neste estudo demonstram o status social destes pacientes: a eczemátide, mais comum nestes grupos; a dermatite das fraldas, que em muitos casos, conforme observamos, ocorria pela ausência de acompanhante para troca constante das fraldas, ou mesmo pela presença de mãe ou parente sem a orientação quanto aos cuidados necessários com a criança; e a xerodermia, geralmente acompanhada de algum grau de desnutrição.

As demais dermatoses, que tiveram número mais significativo de casos, são comuns entre todos os níveis sociais sem distinção, como as lesões melanocíticas e prurigo estrófulo.

As piodermites, dermatite atópica e dermatozoonoses não variaram segundo dados ambulatoriais de clínica privada.?

Para finalizar, é importante ressaltar o quanto as patologias cutâneas são comuns na infância. No entanto, para se conhecer melhor as características epidemiológicas das dermatoses nas crianças internadas, seria necessário um outro estudo com número maior de pacientes, em que, na amostra, além da população internada, fosse também incluído um grupo de crianças da população geral. Assim, seria possível conhecer o comportamento das dermatoses na cidade do Rio de Janeiro, com todas as suas particularidades de condições socioeconômicas e ambientais e, com estes dados, ter uma base melhor para conhecer as diferenças entre estes dois grupos.

\section{Conclusões}

- A prevalência das dermatoses na enfermaria de pediatria do HUPE foi de $83,67 \%$;

- $\quad$ as dermatoses mais comuns (>2\%) foram: nevos melanocíticos, mancha mongoliana, dermatite das fraldas, eczemátide, mancha café com leite, prurigo estrófulo, xerodermia, miliária, dermatite seborreica, nevo hipocrômico, dermatite atópica, celulite, dermatite de contato e alopecia difusa pós-quimioterapia;

- a patologia apresentada pelos pacientes na internação provocou o aumento na prevalência de algumas afecções, como candidíase oral, herpes-zóster, púrpura (petequias e equimoses) e alopecia difusa;

- observamos aumento na frequência dos nevos melanocíticos, eczemátide e piodermites de acordo com a faixa etária estudada e diminuição da mancha mon- 
goliana, dermatite das fraldas e miliária com a idade;

- as doenças infectoparasitárias não apresentaram variações em relação aos dados encontrados na literatura, inclusive de clínicas privadas;

- as demais dermatoses com número mais significativo de casos (nevo melanocítico, mancha mongoliana etc) também não apresentaram variações na sua frequência em relação aos outros estudos;

- $\quad$ em $13,36 \%$ dos pacientes a causa da internação era uma doença estudada pela dermatologia.

\section{Referências}

1. Radbill SX. Pediatric Dermatology: Chronologic Excursions into the Literature - Part I. Pediatrics Dermatology in General Medical Texts. Int J Dermatol. 1987;26(4):250-6.

2. Radbill SX. Pediatric Dermatology: Chronologic Excursions into the Literature - Part II. Dermatology in Pediatric Texts. Int J Dermatol. 1987; 26(5): 324-31.

3. Honig PJ, Burke L. The subspecialty of pediatric dermatology. J Am Acad Dermatol. 1986;15(1):123-6.

4. Caputo RV. Recent advances in pediatric dermatology. In: Rasmussen, J. E. The pediatric clinics of North America. 1983;30(4):735-48.
5. Krowchuk DP, Tunessen WW Jr, Hurwitz S. Pediatric Dermatology Update. Pediatrics. 1992;90(2):259-264.

6. Fuentes ME, Batista M, Melendez S, Artiles AC. Caracteristicas de las dermatosis en Pediatria. Revista Medica Dominicana. 1988;49(3):75-78.

7. Greco P, Cafiero P, Ripoli M, Pierini AM. Proporción de patología dermatológica en la atencion ambulatoria pediátrica. Archivos Argentinos de Dermatologia. 1997;47(2):91-97.

8. Bechelli LM, Haddad N, Pagnano PMG, Tanaka AMU, Zanin LC, Santos MROR, et al. Censo epidemiológico de Lesões e afecções cutâneas em escolares de Ribeirão Preto, SP, Brasil. Archivos Argentinos de Dermatologia. 1990;40(1):45-59.

9. Pizzol JL. Incidência de dermatoses em crianças de zero a seis anos de idade no município de Viana Espírito Santo - no ano de 1985. Anais Brasileiros de Dermatologia. 1988;63(1):15-8.

10. Nascimento LV, Rozo EM, Yarak S, Coimbra SD, Porto J. Prevalência das dermatoses em recém nascidos no berçário de um hospital universitário. Anais Brasileiros de Dermatologia. 1992;67(6):305-7.

11. Mendonça IM, Hernandez OEM, Perdomo JGC. Inquérito epidemiológico das dermatoses numa instituição infantil. Anais da Academia Nacional de Medicina. 1995;155(2):85-8.

12. Minelli L, Minelli HJ. Dermatoses na infância: estudo estatístico de 6.000 casos. Anais Brasileiros de Dermatologia. 1992;67(1):15-8.

13. Sampaio SAP. Patologia da pele e anexos. In: Marcondes E. Pediatria Básica. 8a ed (2). São Paulo: Sarvier; 1991. p. 1638-97

\section{Giane Pereira Giro Teixeira}

Especialista em Dermatologia pela Sociedade Brasileira de Dermatologia.

\section{Alexandre Carlos Gripp}

Serviço de Dermatologia. Hospital Universitário Pedro Ernesto. Faculdade de Ciências Médicas. Universidade do Estado do Rio de Janeiro. Rio de Janeiro, RJ, Brasil. 